\title{
Commentary
}

\section{Some topics in theoretical population genetics: Editorial commentaries on a selection of Marc Feldman's TPB papers}

\author{
Lee Altenberg ${ }^{\mathrm{a}}$, Nicole Creanza ${ }^{\mathrm{b}}$, Laurel Fogarty ${ }^{\mathrm{c}}$, Lilach Hadany ${ }^{\mathrm{d}}$, Oren Kolodny ${ }^{\mathrm{e}}$, Kevin \\ N. Laland ${ }^{\mathrm{f}}$, Laurent Lehmann ${ }^{\mathrm{g}}$, Sarah P. Otto ${ }^{\mathrm{h}}$, Noah A. Rosenberg ${ }^{\mathrm{e}, *}$, Jeremy Van Cleve ${ }^{\mathrm{i}}$, \\ John Wakeley ${ }^{j}$ \\ a Information and Computer Sciences, University of Hawai'i at Mānoa, Honolulu, HI 96822, USA \\ ${ }^{\mathrm{b}}$ Department of Biological Sciences, Vanderbilt University, Nashville, TN 37232, USA \\ ${ }^{\mathrm{c}}$ The UCL Centre for Computation, Mathematics and Physics in the Life Sciences and Experimental Biology, University College London, London, WC1E \\ 6BT, United Kingdom \\ ${ }^{\mathrm{d}}$ Department of Molecular Biology E Ecology of Plants, Tel Aviv University, Tel Aviv, 6997801, Israel \\ e Department of Biology, Stanford University, Stanford, CA 94305, USA \\ ${ }^{\mathrm{f}}$ School of Biology, University of St. Andrews, St. Andrews, Fife KY16 9TH, United Kingdom \\ ${ }^{g}$ Department of Ecology and Evolution, Université de Lausanne CH-1015, Switzerland \\ ${ }^{\mathrm{h}}$ Department of Zoology, University of British Columbia, Vancouver, BC V6T 1Z4, Canada \\ ${ }^{\mathrm{i}}$ Department of Biology, University of Kentucky, Lexington, KY 40506, USA \\ ${ }^{\mathrm{j}}$ Department of Organismic and Evolutionary Biology, Harvard University, Cambridge, MA 02138, USA
}

\section{A R T I C L E I N F O}

\section{Article history:}

Received 22 February 2018

Available online 26 December 2018

\begin{abstract}
A B S T R A C T
This article consists of commentaries on a selected group of papers of Marc Feldman published in Theoretical Population Biology from 1970 to the present. The papers describe a diverse set of population-genetic models, covering topics such as cultural evolution, social evolution, and the evolution of recombination. The commentaries highlight Marc Feldman's role in providing mathematically rigorous formulations to explore qualitative hypotheses, in many cases generating surprising conclusions.
\end{abstract}

(c) 2018 Elsevier Inc. All rights reserved.

\section{Introduction}

As part of this special issue, we are pleased to include brief commentaries on a highlighted subset of articles reported in Theoretical Population Biology by Marc Feldman. The choice of this group of 15 from a bibliography of more than 75 articles was not trivial. Each article in the collection has had significant impact in inspiring future studies, and the earlier papers in the group cover topics that continue to be of great interest many years later. The articles represent the breadth of Marc's theory contributions as much as can be accomplished for a small collection, illustrating themes on "some topics in theoretical population genetics" (Feldman, 1969) that have persisted over many decades of his theoretical work.

-Noah A. Rosenberg

\section{On the evolutionary effect of recombination (1970)}

Selection for linkage modification: I. Random mating populations (1972)

Evolution takes twists and turns that often generate surprising outcomes. Mathematical modeling is critical in such cases, as intuition often fails. One important example is the evolution of sex

\footnotetext{
* Corresponding author.

E-mail address: noahr@stanford.edu (N.A. Rosenberg).
}

and recombination. Intuitively, we might imagine that sex and recombination are advantageous because they generate the genetic variation upon which evolution depends. In a pair of now classic studies, however, Marc Feldman demonstrated that the evolutionary advantages of sex and recombination are not so simple: recombination can decrease variation, recombination can unravel genetic associations built up by past selection, and recombination can be selected against.

Eshel and Feldman (1970) explored the effect of epistasis (nonmultiplicative interactions) on the genetic associations that build up by selection over time between two loci. With directional selection favoring the $a b$ haplotype in an initially $A B$ population, the authors proved that, whenever fitness was "super-multiplicative" (positive epistasis), recombining populations would actually have fewer double mutants than would asexual populations. Recombination actually decreases fitness variation in such cases.

Feldman (1972) went on to prove the remarkable result that increased recombination is never favored in populations at equilibrium under selection. Specifically, after introducing an allele at a third "modifier" gene that alters recombination between two selected loci, only modifier alleles that tighten linkage could invade. This proof, while initially shown for a specific set of fitness regimes, was later shown to hold in a variety of cases (see review 
by Feldman et al. (1996)). Underlying this result is the fact that genetic associations present at equilibrium have been favored by past selection. Breaking apart these associations tends to reduce fitness and select against recombination.

To paraphrase Eshel and Feldman's conclusion, the main aim of this work was to rigorously check commonly held views about the advantages of recombination. These views crumbled under analysis. From these ashes, new modifier models were later built that clarified what had to be added for increased sex and recombination to evolve, such as environmental change, mutation, more complex inheritance (e.g., with non-random mating or non-Mendelian inheritance), or drift alongside selection (Feldman et al., 1996). It is in these contexts that recombination can do more than simply unravel what past selection has built. It is in these contexts that increased sex and recombination can evolve, not because intuition says they should... but because models proved it to be possible.

-Sarah P. Otto

\section{Identity of genes by descent within and between populations under mutation and migration pressures (1972)}

Geographic population structure resulting from limited dispersal is a fundamental aspect of genetic diversity. Measurements of variation within and between populations were first made using gel electrophoresis of proteins in the mid 1960s. With these data in mind, several important theoretical studies of diversity under the action of infinitely-many-alleles mutation were done in the early 1970s. In this article, Nei and Feldman (1972) considered probabilities of identity by descent for pairs of samples taken either within or between populations. They assumed a simple non-equilibrium scenario in which two populations split from an ancestral population at some time in the past and after that exchange migrants at a constant rate. They found an example of the now well studied phenomenon that expected values of withinpopulation pairwise diversity are surprisingly invariant to the migration rate. They further emphasized the slow rate of approach to equilibrium of these probabilities of identity by descent, and hence the propensity for errors in the application of equilibrium formulas to data from any natural populations. However, what stands out even more about this 1972 article is the fact that it includes the full time-dependent analysis of the development of identity probabilities. Nei and Feldman were among the first to do this, certainly the first for this non-equilibrium scenario, and now looking back we may see the entire process of pairwise times to common ancestry in a migration-structured population model clearly embedded in their analysis.

\section{-John Wakeley}

\section{Models for cultural inheritance I. Group mean and within group variation (1973) \\ Cultural and biological evolutionary processes, selection for a trait under complex transmission (1976)}

From the early 1970s, Marc Feldman (together with Luca Cavalli-Sforza) began modeling cultural inheritance, cultural change over time, and the coevolutionary interactions between genes and culture. This work provided the formal foundations of the now well-established fields of cultural evolution and geneculture coevolution. Important early papers were Cavalli-Sforza and Feldman (1973) and Feldman and Cavalli-Sforza (1976). It is difficult to overstate the importance of these papers, in which much of the formal machinery for the mathematic modeling of culture is introduced. Methods are presented for modeling the cultural inheritance of both discrete and continuous phenotypic variation, including uniparental and biparental transmission as well as wider influences from the previous or current generation, and for investigating the coevolution of genes and culture through tracking alternative genotype-phenotype combinations (phenogenotypes), all under a variety of selection regimes. These analyses provided the foundations for Feldman and Cavalli-Sforza's (1981) classic monograph Cultural Transmission and Evolution, and had a lasting influence on many researchers, notably anthropologists Robert Boyd and Peter Richerson, and their students.

The tense and fractious context in which these works were introduced were the nature-nurture and human sociobiology debates. Feldman and Cavalli-Sforza's analysis cut through the polemics to provide practical tools with which researchers could ask scientific questions about these complex issues (e.g. Can selection on a culturally determined character cause changes in gene frequencies?). The emerging body of theory has been used in a variety of ways (see the comments by Creanza, Lehmann, Fogarty, and Kolodny, below), including partitioning the variance in behavioral and personality traits into a variety of components including a transmitted cultural component, addressing some very general questions about the adaptive advantages of complex forms of phenotypic plasticity, exploring the diverse processes responsible for cultural change, and investigating specific cases in which there is an interaction between a cultural trait and some genetic variation that may influence, or be influenced by, its prevalence. Almost 50 years later, the field of cultural evolution has never been more vibrant, with a new international society (https:// culturalevolutionsociety.org) formed in 2017, with its own conferences and a journal planned. That vigor owes a huge debt to Marc Feldman's seminal contribution.

-Kevin N. Laland

\section{Darwinian selection and "altruism" (1978) \\ Theories of kin and group selection: a population genetics per- spective (1980)}

During Marc Feldman's seventy-fifth birthday celebration, many of his students commented on his talent for cultivating rigorous thinking and an appreciation of mathematical analysis. One of the areas in evolutionary biology most in need of Feldman's precision and clarity was the evolution of altruism. Until the publication in 1964 of Bill Hamilton's mathematical theory of kin selection and inclusive fitness, research on the evolution of altruism had been mostly confined to verbal theories proffered by ethologists such as Konrad Lorenz and V. C. Wynne-Edwards. These verbal theories advocated that natural selection can favor cooperative behavior simply because such behavior can increase the persistence of groups or populations, and they ignored the individual-level fitness costs of cooperation.

A key feature of Hamilton's theory was its simplicity, which is captured in his eponymous rule that states that cooperation evolves when its fitness costs are exceeded by the benefits weighted by the "genetic relatedness" between the donor and recipient of cooperation. Though Hamilton's theory eventually became one of the "central dogmas of the modern theory of evolution" (Eshel and Feldman, 2001), the 1964 paper derived its result using a fitness maximization procedure, which had been proven in the same year by P. A. P. Moran to be unreliable in the case of multiple loci. Subsequent work on kin selection in the 1970s built on the maximization argument despite its known weakness. CavalliSforza and Feldman (1978) stepped into this void and provided population-genetic models of kin selection among close relatives. Cavalli-Sforza and Feldman assumed that the fitness costs and benefits of altruistic behavior interact either multiplicatively or additively. They found that the conditions for the initial increase of altruism and its resistance to invasion by nonaltruism corresponded to Hamilton's rule when fitness is additive but not multiplicative. This result provided a firm population-genetic footing to both Hamilton's rule and also to the restrictions required for the rule to hold.

Even as kin selection gained traction in the 1970s, evolutionary theorists such as Ilan Eshel, D. S. Wilson, and George Price studied 
models of group selection that properly included both individuallevel costs and group-level benefits. The backlash against the ideas of Lorenz and Wynne-Edwards generated and continues to generate confusion about the fundamental mechanisms that allow cooperation to evolve in these models. From the vantage point of dynamic population-genetic models, Uyenoyama and Feldman (1980) provided one of the first comprehensive and synthetic perspectives about group selection, its mechanisms, and its relationship to kin selection.

Uyenoyama and Feldman (1980) first grounded its discussion of group selection by noting that Sewall Wright already had studied many of the fundamental issues in his work on the "shifting balance theory." Specifically, Uyenoyama and Feldman noted that Wright had understood that "random processes such as drift... are capable of producing the interdemic variation of phenotype essential for group selection." Uyenoyama and Feldman also emphasized that group selection involves both differential migration (more cooperative groups are more productive and produce more migrants) and differential extinction (more cooperative groups are less likely to go extinct). It is the combination of these mechanisms, (i) interdemic variation due to drift (or other forces) that yields some groups with more cooperators than others and (ii) increased production of individuals by more cooperative groups, that allows cooperation to evolve under group selection. Much theoretical work in the 1990s and 2000s revisited the same issues regarding the mechanisms of group selection and their relationship to kin selection; even placed among the best examples of that more recent work, Uyenoyama and Feldman (1980) still captures the most important insights with undiminished clarity.

-Jeremy Van Cleve

Modifiers of mutation-rate: a general reduction principle (1986) A general reduction principle for genetic modifiers of recombination (1986)

Feldman (1972) (see the comment by Otto, above) initiated the spectral analysis of the invasion dynamics of selectively neutral genes that modify the recombination rates at other genes undergoing natural selection, and found that only modifiers which reduced the rate of recombination could invade a population at equilibrium (the Reduction Principle). Feldman, as my doctoral advisor, introduced me to the subject by posing a very general conjecture (1980, personal communication) on the general existence of viability-analogous Hardy-Weinberg (VAHW) modifier polymorphisms. These are equilibria in infinite diploid populations in which each modifier allele produces the same mean value $m^{*}$ for a parameter that enters linearly in the dynamics of the genes under natural selection, where those genes would be at a stable equilibrium were the modifier fixed for an allele that produced the same value $m^{*}$, and those genes are in linkage equilibrium with the modifier locus.

Feldman's conjecture formed the kernel of my dissertation (Altenberg, 1984), which proved his VAHW conjecture, as well as the reduction principle for arbitrary genetic systems, numbers of haplotypes, and selection regimes, but with the constraint that the modifiers be either tightly linked to the major loci, or produce perfect transmission of the haplotypes under selection (these constraints would be overcome eventually in Altenberg (2009)). The internal stability of the entire modifier-major locus polymorphism could be analyzed only for special cases.

Liberman and Feldman were able to completely solve these general questions for modifiers of recombination rates (Liberman and Feldman, 1986b) and mutation rates (Liberman and Feldman, 1986a), in models with two alleles at one or two major loci, but with unlimited numbers of modifier alleles. They confirmed that VAHW equilibria always existed, and showed that (1) a new modifier allele could invade a VAHW polymorphism if and only if it reduced the mutation or recombination rate for the major loci, for arbitrary linkage between modifier and major loci; and (2) the VAHW polymorphism was internally stable when linkage was loose enough to the modifier locus and - most significantly - when the fidelity values $(1-m)$ produced by each diploid modifier locus had exactly the same spectral properties as are required for the stability of a single-locus, multiple-allele viability polymorphism.

As inspection of their proofs will reveal, the papers are masterpieces of mathematical analysis involving the intricate use of characteristic polynomials and spectral analysis. Their work shows that in many fundamental respects, fidelity of replication behaves mathematically exactly as does viability. The "viability analogy" exhibited by genes that control genetic information transmission is not mere metaphor, but a deep mathematical phenomenon.

-Lee Altenberg

\section{Waiting with and without recombination: the time to produc- tion of a double mutant (1998)}

The rate at which asexual populations cross fitness valleys (2009)

Complex adaptations - ones that require multiple mutations - are a key to the understanding of most phenotypes and behaviors encountered in nature. The fact that recombination allows the incorporation of beneficial mutations that appeared in separate individuals into a single genome, potentially facilitating the appearance of complex adaptations, is considered a major evolutionary advantage of sexual reproduction.

Christiansen et al. (1998) investigated the fundamental question of how long it would take for two mutations necessary for a new complex trait to appear in one individual, where each mutation is beneficial or neutral. In this study, the authors provide a thorough and meticulous analysis of the waiting time to the emergence of a double mutant, considering various factors, such as population size, mutation rate, selection advantage of single mutants, stochastic effects, and in particular, recombination. The authors provide several alternative analysis techniques that best suit the assumptions, and derive approximations for the expected waiting time. The comprehensive analysis highlights the factors that have a major effect on the waiting time, and reveal that while recombination always facilitates the appearance of double mutants, the effect is negligible in large populations, and minor in smaller populations.

Weissman et al. (2009) continued the work on complex adaptation in the intriguing case of "valley crossing," where each single mutation is deleterious. In such cases, adaptation can take an extremely long time, and the population can even be trapped in a local fitness maximum. The 2009 work concentrated on the case of asexual populations and investigated the rate of valley crossing under a wide range of parameters. They showed that valley crossing can be quite easy in large populations, even in the case of a wide valley requiring multiple mutations. In contrast, deep valleys (i.e., ones with a strong selective disadvantage) might limit complex adaptation in small populations. A subsequent study of Weissman et al. (2010) extended the analysis to sexual populations, showing that recombination can have a strong and nonmonotonic effect on the rate of valley crossing. They found that low recombination can increase the rate by orders of magnitude, while high recombination can in fact lower the rate of valley crossing through breakdown of the complex adapted genotype.

Together, these studies show that the actual dynamics of producing complex adaptation can be unexpectedly complex. As in many other cases (see the comments by Otto and Altenberg, above), the effect of recombination is surprising and intuition is not enough to predict it-detailed models are required to reveal the conditions where the advantage of recombination in complex adaptation is significant.

-Lilach Hadany 


\section{Cultural niche construction and the evolution of small family size (2004)}

In 1929, the demographer Warren Thompson noticed a pattern: as countries developed and industrialized, the death rates in those countries fell, with a drop in birth rates closely following. From an evolutionary perspective, a reduced birth rate appears to be counterintuitive and unstable, since the preference or ability to have more offspring instead of fewer should be overrepresented in the next generation and thus should easily spread through a population. In this light, the repeated evolution of small family sizes in human populations is a paradox: it is unclear how a small family-size strategy could persist, since an alternative strategy of having many offspring should, by definition, increase fitness. Ihara and Feldman (2004) proposed an elegant explanation to this dilemma by considering the effect of oblique cultural transmission. The evolutionary puzzle implicitly hinges on children adopting the family-size strategy of their parents. However, Ihara and Feldman considered an incisive solution: what if individuals with fewer offspring are more likely to pass on their cultural traits? These individuals could have different social roles such as teachers or managers, could be perceived to have more prestige or success in the community, or could simply have more time to transmit their beliefs. Thus, an individual that leaves more offspring does not guarantee that any of those offspring will have large families. This simple idea effectively unlinks the biological and cultural underpinnings of family size, providing a possible resolution to the evolutionary paradox.

-Nicole Creanza

\section{Evolution of social learning: a mathematical analysis (2004)}

Should individual behavior be determined innately, acquired by trial-and-error (individual learning) or copied from other individuals in the population (social learning)? Previous mathematical models addressing the evolutionary stability of these competing strategies have mainly focused on the tension between individual and social learning. Wakano et al. (2004) was the first to fully take into account the three strategies, innateness, individual learning and social learning in a situation where the environment can change at short and long intervals, or stochastically. The paper shows that depending on the rate of environmental change, there is a critical level of environmental constancy, above which innate individuals are favored by selection, and below which they are eliminated with individual and social learners remaining in a protected polymorphism. By establishing benchmark conditions under which both individual and social learning are favored by selection, these results deepen the understanding of biological situations in which learning can evolve.

-Laurent Lehmann

Rates of cultural change and patterns of cultural accumulation in stochastic models of social transmission (2011)

One topic of long-standing interest in cultural evolution has been the effect of modes of cultural transmission, or how information spreads from one person to another, on the rate and direction of cultural evolution (see the comment by Laland, above). It had been argued that certain transmission modes might lead to rapid cultural change (a pro-novelty bias, for example) where others might slow cultural evolution considerably (e.g. conformist transmission). Verbal arguments linking the mode of transmission to the rate of cultural change had been used to suggest that certain types of learning might have been prevalent at particular times in human history or in particular populations. Separately, disagreements had arisen over the possible role of population size in determining the level of cultural complexity within a population - with theoretical analyses pointing to an important role for population size, and many statistical analyses pointing, instead, to a role for environmental change.
In this paper, Aoki et al. (2011) linked innovation, modes of transmission, and, crucially, population size, by making two important modeling decisions. First, they revealed that synchronous updating assumptions give an inflated importance to horizontal and oblique transmission, and by relaxing that assumption they were able to fairly compare the effects of oblique transmission and vertical transmission. Second, in addition to the commonly modeled random oblique transmission, they investigated a broader range of transmission modes including learning from a teacher, learning with a pro-novelty bias, and conformist learning.

Some of the results confirmed intuition. For example, they showed that conformist transmission led to slow rates of cultural change and pro-novelty bias to rapid evolution. In other areas, the model defied expectations, particularly in relation to cultural heterogeneity (or how one individual's cultural repertoire differs from another's). Conformist transmission, for example, led to high heterogeneity where individuals continued to innovate without spreading new traits. The model also strengthened the argument that population size could play an important role in cultural evolution, but stressed that this relationship depended on the mode of cultural transmission employed, adding a previously unappreciated layer of nuance.

Understanding how likely an innovation is to spread in a population, and how rapidly this may happen is crucial to the study of cultural complexity. The model described by Aoki et al. (2011) was a significant step forward in understanding cultural complexity, the rate of cultural evolution, and their relationships to innovation and transmission.

-Laurel Fogarty

The role of cultural transmission in human demographic change: an age-structured model (2013)

Two great revolutions have characterized human demography: the Neolithic revolution, in which the switch from huntergatherer societies to agriculturalists was characterized by a change in population age structure and a shift to high population growth, and the second demographic transition, observed in developed regions such as western Europe and the United States, reflected by a shift to low fertility and low mortality. The latter, in particular, is hard to explain from a perspective of behavioral ecology: it is achieved via the spread of practices such as birth control, an intentional reduction of reproductive output. Despite the complexity of the processes that brought about these demographic shifts and the ensuing changes in population age structure, Fogarty et al. (2013) provide in this study a tour de force of the way in which a model with few variables may explain profound phenomena. They provide an analytical treatment that describes the fate of a population in which a cultural trait that influences fertility, survival, or both, may appear and spread. Their model shows how different transmission dynamics of this trait-vertical, horizontal, or oblique learning-would influence the population's demography. This analysis suggests an elegant explanation to the second demographic transition, demonstrating how some biological phenomena make no sense except in the light of cultural evolution.

-Oren Kolodny

\section{References}

Altenberg, L., 1984. A generalization of theory on the evolution of modifier genes (Ph.D. thesis). Stanford University, Stanford, CA.

Altenberg, L., 2009. The evolutionary reduction principle for linear variation in genetic transmission. Bull. Math. Biol. 71, 1264-1284.

Aoki, K., Lehmann, L., Feldman, M.W., 2011. Rates of cultural change and patterns of cultural accumulation in stochastic models of social transmission. Theor. Popul. Biol. 79, 192-202.

Cavalli-Sforza, L., Feldman, M.W., 1973. Models for cultural inheritance I. Group mean and within group variation. Theor. Popul. Biol. 4, 42-55. 
Cavalli-Sforza, L.L., Feldman, M.W., 1978. Darwinian selection and “altruism”. Theor. Popul. Biol. 14, 268-280.

Christiansen, F.B., Otto, S.P., Bergman, A., Feldman, M.W., 1998. Waiting with and without recombination: the time to production of a double mutant. Theor. Popul. Biol. 53, 199-215.

Eshel, I., Feldman, M.W., 1970. On the evolutionary effect of recombination. Theor. Popul. Biol. 1, 88-100.

Eshel, I., Feldman, M.W., 2001. Individual selection and altruistic relationships: The legacy of W. D. Hamilton. Theor. Popul. Biol. 59, 15-20.

Feldman, M.W., 1969. Some topics in theoretical population genetics (Ph.D. thesis). Stanford University, Stanford, CA.

Feldman, M.W., 1972. Selection for linkage modification: I. Random mating populations. Theor. Popul. Biol. 3, 324-346.

Feldman, M.W., Cavalli-Sforza, L.L., 1976. Cultural and biological evolutionary processes, selection for a trait under complex transmission. Theor. Popul. Biol. 9, 238-259.

Feldman, M.W., Cavalli-Sforza, L.L., 1981. Cultural Transmission and Evolution. Princeton University Press, Princeton, NJ.

Feldman, M.W., Otto, S.P., Christiansen, F.B., 1996. Population genetic perspectives on the evolution of recombination. Annu. Rev. Genet. 30, 261-295.
Fogarty, L., Creanza, N., Feldman, M.W., 2013. The role of cultural transmission in human demographic change: an age-structured model. Theor. Popul. Biol. 88 68-77.

Ihara, Y., Feldman, M.W., 2004. Cultural niche construction and the evolution of small family size. Theor. Popul. Biol. 65, 105-111.

Liberman, U., Feldman, M.W., 1986a. Modifiers of mutation-rate: a general reduction principle. Theor. Popul. Biol. 30, 125-142.

Liberman, U., Feldman, M.W., 1986b. A general reduction principle for genetic modifiers of recombination. Theor. Popul. Biol. 30, 341-371.

Nei, M., Feldman, M.W., 1972. Identity of genes by descent within and between populations under mutation and migration pressures. Theor. Popul. Biol. 3, 460465.

Uyenoyama, M., Feldman, M.W., 1980. Theories of kin and group selection: a population genetics perspective. Theor. Popul. Biol. 17, 380-414.

Wakano, J.Y., Aoki, K., Feldman, M.W., 2004. Evolution of social learning: a mathematical analysis. Theor. Popul. Biol. 66, 249-258.

Weissman, D.B., Desai, M.M., Fisher, D.S., Feldman, M.W., 2009. The rate at which asexual populations cross fitness valleys. Theor. Popul. Biol. 75, 286-300.

Weissman, D.B., Feldman, M.W., Fisher, D.S., 2010. The rate of fitness-valley crossing in sexual populations. Genetics 186, 1389-1410. 